\begin{abstract}
This study explored the development of cool and hot EF skills across early childhood. Children 4.5- to 5.5-years-old $(N=80)$ completed performance-based assessments of cool EF (inhibition and working memory), hot EF (affective decision making and delay of gratification) at three time points across 12 months. Cool EF task performance was consistently correlated across early childhood, but hot EF task performance was not. Performance on cool EF tasks showed significant improvements over early childhood, but performance on hot EF tasks did not. During early childhood performance on delay of gratification and affective decision making tasks may therefore be unrelated and show limited sensitivity to improvement.
\end{abstract}

Key words. Executive function, hot executive function, cognitive development. 


\section{Associations between and development of cool and hot executive functions across early childhood}

Researchers are increasingly referring to cool and hot executive function (EF). Whereas cool EF refers to the more purely cognitive skills (e.g. inhibition, working memory), hot EF denotes the more affective EF processes (Zelazo \& Müller, 2002). The most commonly assessed hot EF skills are delay of gratification (Kochanska, Murray, Jacques, Koenig, \& Vandegeest, 1996), an ability that is often considered reflective of effortful control (Allan \& Lonigan, 2011), and affective decision making (Kerr \& Zelazo, 2004; Zelazo \& Müller, 2002). However, understanding of the organisation and development of hot EF lags behind that of cool EF.

Research attempting to identify whether cool EF and hot EF represent distinct constructs in early childhood has resulted in contradictory findings. Although some studies have found cool and hot domains of EF are not correlated in young children, supporting a multidimensional model (Di Norcia, Pecora, Bombi, Baumgartner, \& Laghi, 2014), other research has suggested a uni-dimensional model incorporating cool and hot skills (Allan \& Lonigan, 2011). Further contributing to the current confusion concerning the structure of hot EF is emerging research that indicates skills considered to reflect hot EF are not strongly associated with one another. Some research has found delay of gratification and affective decision making to be moderately correlated in children 3- to 5-years-old, once chronological and mental age were taken into account (-.24; Hongwanishkul, Happaney, Lee, \& Zelazo, 2005). In contrast, other research has found a lack of an association between these abilities in 3- to 6-year-old children (Poland et al., 2016).

Limited studies have considered the development of a range of hot EF skills. Substantial gains in cool EF are demonstrated between 3- and 6-years-of-age (Anderson, 1998). 
There is some evidence that four-year-olds have been found to outperform 3-year-olds on hot delay of gratification tasks (Carlson, Claxton, \& Moses, 2013) and 4- and 5-year-olds have been found to outperform 3-year-olds on hot gambling tasks (Hongwanishkul et al., 2005; Kerr \& Zelazo, 2004). These studies, though, are cross-sectional. Early childhood is an important period in the development of EF, reflecting a maturation of the prefrontal cortex (Anderson, 1998), consequently important changes in EF task performance may be missed by crosssectional research. Cool EF may undergo rapid advancement during early childhood, but hot EF may follow a more protracted developmental course. Indeed, improvements in affective decision making and delay of gratification (hot EF) have been found to be more gradual, with more marked changes occuring around 14- to 15-years-old (Prencipe et al., 2011).

This preliminary study therefore explored the association between and changes in performance on cool and hot EF tasks during an important period in cognitive development; early childhood. Four- and 5-year-olds completed a battery of cool and hot EF tasks at three time points: initial recruitment (T1), 6 months later (T2) and 12 months later (T3). It was hypothesised that cool EF task performance would be correlated and as would hot EF task performance. It was hypothesised that improvements on measures of cool EF, but not hot EF, would be demonstrated across early childhood.

\section{Method}

\section{Participants}

Eighty typically-developing children (40 boys) from two mainstream primary schools that were comparable in the percentage of pupils receiving free school meals $(26.6 \%$ and $24.7 \%)$ participated. There were two cohorts (mean ages reported in Table 1): 4-year-olds ( $N$ 
$=48)$ and 5-year-olds $(N=32)$. At T2 73 children were followed up (9\% attrition) and at T3 72 children were followed up (1\% attrition).

\section{Measures and Procedure}

At each time point children completed performance-based assessments of EF.

\section{Cool EF Tasks}

Go/No-Go - Inhibition (Simpson \& Riggs, 2006). Children were required to press a button on a response pad to catch the fish (Go trials), and withhold pressing the button to avoid catching the sharks (No-Go trials). There were 6 practice trials ( 3 Go/3 No-Go trials) and 40 test trials (30 Go/10 No-Go trials). The proportion of correct No-Go trials was measured.

Digit Span Backwards - Working Memory (WISC-III; Wechsler, 1991). Children were instructed to recall number sequences in reverse order (from 2 to 8 digits). One point was awarded for each correct trial. Scores ranged from 0 to 30 .

\section{Hot EF Tasks}

Children's Gambling Task - Affective decision making (CGT; For a description of this task see Poland, Monks, \& Tsermentseli, 2016). Children selected cards from two decks, which when turned revealed happy faces, corresponding to the number of beads won, and sad faces, representing the number of beads lost. One deck was advantageous and resulted in a net win of 5 beads per 10 cards, whereas the other deck was disadvantageous and resulted in a net loss of 5 beads per 10 cards. There were 6 demonstration trials and 50 test trials. The proportion of advantageous to disadvantageous decisions across the last three trial blocks was assessed. Scores ranged from -1 (all disadvantageous decisions) to +1 (all advantageous decisions).

Gift Wrap - Delay of Gratification (Kochanska et al., 1996). The child was instructed not to peek while the researcher pretended to wrap a gift in a standardised manner for 60 
seconds. Two points were awarded if the child did not turn around, 1 point if they peeked over their shoulder and 0 points if they turned around completely.

\section{Results}

Descriptive statistics are reported in Table 1. Go/No-Go and Gift Wrap scores deviated from a normal distribution across time points. All other variables followed an approximately normal distribution across time points. Due to the relatively large sample size and the fact the deviation from normality was minimal parametric tests were still carried out (Little, 2013).

Correlations revealed that Digit Span and Go/No-Go performance were positively and significantly related across time points. Gift Wrap performance was positively related to Go/No-Go performance at all three time points and Digit Span performance at T1 and T2, but was unrelated to CGT performance at all three time points. Correlations are reported in Table 2.

To examine improvements on EF tasks across early childhood mixed ANOVAs were carried out with time (T1, T2, T3) as the within-subject factor and cohort (4- and 5-year-olds) as the between-subject factor. Bonferroni correction was applied to follow-up analyses.

Go/No-Go. There was a significant effect of time, $F(1.84,128.60)=3.56, p=.04, \mathrm{y}_{\mathrm{p}}{ }^{2}$ $=.05 . \mathrm{Go} / \mathrm{No}$-Go performance at T3 was significantly greater than at T2 $(p=.02)$, but did not differ between T1 and T2 $(p=.12)$. There was no significant cohort effect, $F(1,71)=.01, p=$ $.91, \mathrm{y}_{\mathrm{p}}^{2}=<.001$, or interaction between time and cohort, $F(1.84,128.60)=.76, p=.46, \mathrm{y}_{\mathrm{p}}{ }^{2}=$ .01 . 
Digit Span. There was a significant effect of time, $F(2,140)=23.05, p<.001, \mathrm{y}_{\mathrm{p}}{ }^{2}=$ .25. Digit Span performance was significantly greater at T2 than T1 $(p=.02)$ and T3 compared to $\mathrm{T} 2(p<.001)$. The effect of cohort, $F(1,70)=10.40, p=.002, \mathrm{y}_{\mathrm{p}}{ }^{2}=.13$, and interaction between time and cohort were significant, $F(2,140)=4.39, p=.01, \mathrm{y}_{\mathrm{p}}^{2}=.06 .5$-year-olds performed significantly better than 4-year-olds $(p=.002)$, but 4-year-olds showed a steeper increase.

CGT. The effect of time was not significant, $F(2,140)=.25, p=.78, \mathrm{y}_{\mathrm{p}}^{2}=.004$, but the effect of cohort was marginally significant, $F(1,70)=3.53, p=.06, \mathrm{y}_{\mathrm{p}}^{2}=.05 .4$-year-olds performed worse than 5-year-olds $(p=.06)$. There was no significant interaction, $F(2,140)=$ $.06, p=.94, \mathrm{y}_{\mathrm{p}}^{2}=.001$.

Delay of Gratification. The effect of time was not significant, $F(2,140)=.24, p=$ $.78, \mathrm{y}_{\mathrm{p}}{ }^{2}=.003$. There was a significant effect of cohort, $F(1,70)=9.98, p=.002, \mathrm{y}_{\mathrm{p}}^{2}=.13 .4-$ year-olds showed poorer performance compared to 5-year-olds $(p=.002)$. There was no significant interaction, $F(2,140)=.37, p=.69, \mathrm{y}_{\mathrm{p}}^{2}=.01$.

\section{Discussion}

The current study examined the association between and changes in performance on cool and hot EF tasks during early childhood. Strengths of this study include its longitudinal design and focus on EF tasks during early childhood. Although performance on cool EF tasks was consistently related across early childhood, performance on hot EF tasks was not related, partially supporting the hypothesis. Performance on Gift Wrap and CGT may therefore not be the result of the same underlying factor. Indeed, the Gift Wrap task is often proposed to assess effortful control (Allan \& Lonigan, 2011), which may explain the correlation between Gift 
Wrap and Go/No-Go performance. CGT performance may represent a distinct cognitive process that is not reflective of EF, cool or hot (Toplak, Sorge, Benoit, West, \& Stanovich, 2010). Future research should focus on elucidating the organisation of hot EF and whether these skills represent a dissociable construct.

In partial support of the hypothesis, improvements in cool (but not hot) EF task performance was evident across early childhood, but in line with prior studies (Kerr \& Zelazo, 2002), 5-year-olds out-performed 4-year-olds on hot EF measures. Findings should be interpreted in light of study limitations including the small sample size and that some tasks may not have been sensitive to developmental changes in EF ability across early childhood. Performance on the Go/No-Go and Gift Wrap tasks, for example, were consistently high across time points for all cohorts, which may indicate a ceiling effect. Future attention needs to be directed to the development of hot EF measures that can be used longitudinally. 


\section{References}

Allan, N. P., \& Lonigan, C. J. (2011). Examining the dimensionality of effortful control in preschool children and its relation to academic and socioemotional indicators. Developmental Psychology, 47, 905-915. doi:10.1037/a0023748

Anderson, V. (1998). Assessing executive functions in children : Biological, psychological, and developmental considerations. Assessment of Attention and Executive Function., 8, 319-349. doi:10.1080/713755568

Carlson, S. M., Claxton, L. J., \& Moses, L. J. (2013). The Relation between executive function and theory of mind is more than skin deep. Journal of Cognition and Development, 16, 186-197. doi:10.1080/15248372.2013.824883

Di Norcia, A., Pecora, G., Bombi, A. S., Baumgartner, E., \& Laghi, F. (2015). Hot and cool inhibitory control in Italian toddlers: Associations with social competence and behavioral problems. Journal of Child and Family Studies, 24, 909-914. doi:10.1007/s10826-014-9901-z

Hongwanishkul, D., Happaney, K. R., Lee, W. S. C., \& Zelazo, P. D. (2005). Assessment of hot and cool executive function in young children: Age-related changes and individual differences. Developmental Neuropsychology, 28, 617-44. doi:10.1207/s15326942dn2802_4

Kerr, A., \& Zelazo, P. D. (2004). Development of "hot" executive function: the children's gambling task. Brain and Cognition, 55, 148-157. doi:10.1016/S0278-2626(03)00275-6 
Kochanska, G., Murray, K., Jacques, T. Y., Koenig, A. L., \& Vandegeest, K. A. (1996). Inhibitory control in young children and its role in emerging internalization. Child Development, 67, 490-507. doi:10.2307/1131828

Little, T. D. (2013). The Oxford Handbook of Quantitative Methods: Volume 1 Foundations. Oxford: Oxford University Press.

Poland, S. E., Monks, C. P., \& Tsermentseli, S. (2016). Cool and hot executive function as predictors of aggression in early childhood: Differentiating between the function and form of aggression. British Journal of Developmental Psychology, 34, 181-197. doi:10.1111/bjdp.12122

Prencipe, A., Kesek, A., Cohen, J., Lamm, C., Lewis, M. D., \& Zelazo, P. D. (2011). Development of hot and cool executive function during the transition to adolescence. Journal of Experimental Child Psychology, 108, 621-637. doi:10.1016/j.jecp.2010.09.008

Simpson, A., \& Riggs, K. J. (2006). Conditions under which children experience inhibitory difficulty with a "'button-press" go/no-go task. Journal of Experimental Child Psychology, 94, 18-26. doi:10.1016/j.jecp.2005.10.003

Toplak, M. E., Sorge, G. B., Benoit, A., West, R. F., \& Stanovich, K. E. (2010). Decisionmaking and cognitive abilities: A review of associations between Iowa Gambling Task performance, executive functions, and intelligence. Clinical Psychology Review, 30, 562-581. doi:10.1016/j.cpr.2010.04.002

Wechsler, D. (1991). Wechsler Intelligence Scale for Children-Third Edition. San Antonio, TX: Psychological Corporation. 
Zelazo, P. D., \& Müller, U. (2002). Executive function in typical and atypical development. In U. Goswami (Ed.), Handbook of Childhood Cognitive Development (pp. 445-470). Oxford: Blackwell Publising Ltd. 
Table 1. Descriptive statistics for EFs across early childhood

\begin{tabular}{|c|c|c|c|c|c|c|c|c|c|c|c|c|c|c|c|c|c|c|}
\hline & \multicolumn{6}{|c|}{$\mathrm{T} 1$} & \multicolumn{6}{|c|}{$\mathrm{T} 2$} & \multicolumn{6}{|c|}{ T3 } \\
\hline & \multicolumn{2}{|c|}{ All } & \multicolumn{2}{|c|}{ 4-year-olds } & \multicolumn{2}{|c|}{ 5-year-olds } & \multicolumn{2}{|c|}{ All } & \multicolumn{2}{|c|}{ 4-year-olds } & \multicolumn{2}{|c|}{ 5-year-olds } & \multicolumn{2}{|c|}{ All } & \multicolumn{2}{|c|}{ 4-year-olds } & \multicolumn{2}{|c|}{ 5-year-olds } \\
\hline & $M$ & $S D$ & $M$ & $S D$ & $M$ & $S D$ & $M$ & $S D$ & $M$ & $S D$ & $M$ & $S D$ & $M$ & $S D$ & $M$ & $S D$ & $M$ & $S D$ \\
\hline Age & 58.80 & 6.66 & 54.23 & 3.27 & 65.66 & 3.99 & 64.56 & 7.20 & 59.38 & 3.86 & 71.58 & 3.95 & 71.36 & 7.17 & 66.12 & 3.73 & 78.29 & 3.99 \\
\hline Inhibition & .82 & .22 & .81 & .23 & .84 & .20 & .80 & .26 & .80 & .26 & .80 & .25 & .86 & .17 & .87 & .15 & .85 & .19 \\
\hline \multicolumn{19}{|l|}{ Working } \\
\hline Memory & 1.25 & 1.44 & .75 & 1.21 & 2.0 & 1.44 & 1.73 & 1.48 & 1.24 & 1.41 & 2.39 & 1.33 & 2.57 & 1.55 & 2.44 & 1.50 & 2.74 & 1.61 \\
\hline ADM & -.03 & .44 & -.07 & .46 & .04 & .42 & -.07 & .41 & -.12 & .38 & .004 & .44 & -.06 & .42 & -.12 & .33 & .03 & .52 \\
\hline Delay & 1.43 & .80 & 1.30 & .86 & 1.63 & .66 & 1.41 & .81 & 1.19 & .86 & 1.71 & .64 & 1.46 & .73 & 1.29 & .75 & 1.68 & .65 \\
\hline
\end{tabular}

Note. $M=$ mean, $S D=$ standard deviation, ADM = affective decision making, Delay = delay of gratification. 
Table 2. Correlations between cool and hot EF skills across time points

\begin{tabular}{|c|c|c|c|c|c|c|c|c|c|c|c|c|c|}
\hline & 1. & 2. & 3. & 4. & 5. & 6. & 7. & 8. & 9. & 10. & 11. & 12. & 13. \\
\hline 1.T1.Age & - & .06 & $.41 * * *$ & .13 & $.19 \dagger$ & -.04 & $.34 * *$ & .14 & $.28 * *$ & -.11 & $.21^{*}$ & $.31 * *$ & .19 \\
\hline 2. T1 Inhib & & - & $.33 * *$ & .11 & $.43 * * *$ & $.72 * * *$ & $.35 * *$ & -.03 & $.48 * * *$ & $.54 * *$ & $.37 * * *$ & .18 & $.39 * * *$ \\
\hline 3. T1 WM & & & - & -.04 & .16 & $.35^{* *}$ & $.60 * * *$ & -.12 & $.32 * *$ & .02 & $.33 * * *$ & .15 & .22 \\
\hline 4. T1 ADM & & & & - & -.04 & -.06 & -.14 & $.19 \dagger$ & -.18 & -.04 & -.13 & $.19 \dagger$ & -.07 \\
\hline 5. T1 Delay & & & & & - & $.45 * * *$ & $.34 * *$ & -.08 & $.53 * * *$ & $.30 * *$ & $.32 * *$ & .17 & $.36 * * *$ \\
\hline 6. T2 Inhib & & & & & & - & $.37 * *$ & .08 & $.47 * * *$ & $.51 * * *$ & $.43 * * *$ & .06 & $.31 * *$ \\
\hline 7. T2 WM & & & & & & & - & .004 & $.34 * *$ & $.22 *$ & $.68 * * *$ & .08 & $.32 * *$ \\
\hline 8.T2 ADM & & & & & & & & - & -.06 & .17 & -.07 & $.21^{*}$ & $.20^{*}$ \\
\hline 9. T2 Delay & & & & & & & & & - & $.34 * *$ & $.35^{* *}$ & .11 & $.40 * * *$ \\
\hline 10. T3 Inhib & & & & & & & & & & - & $.25^{*}$ & .07 & $.41 * * *$ \\
\hline 11. T3 WM & & & & & & & & & & & - & $.19 \dagger$ & .12 \\
\hline 12. T3 ADM & & & & & & & & & & & & - & .06 \\
\hline 13. T3 Delay & & & & & & & & & & & & & - \\
\hline
\end{tabular}


Note. $\mathrm{VA}=$ verbal ability. Inhib $=$ inhibition. $\mathrm{WM}=$ working memory. $\mathrm{ADM}=$ affective decision making. Delay $=$ delay of gratification. $* p<$ $.05, * * p<.01, * * * p<.001,+p<.06$ 